\title{
Patient Monitoring System
}

\author{
Chanakya Mothukuri ${ }^{1}$, K. CH. Prathap Kumar. $\mathbf{M}^{2}$ \\ ${ }^{1}$ Corresponding Author \\ Department Of Electronics and Communication Engineering \\ CVSR College of Engineering, Hyderabad, India \\ chanakya@gmail.com \\ ${ }^{2}$ Assistant Professor \\ Department Of Electronics and Communication Engineering \\ CVSR College of Engineering, Hyderabad, India \\ chaithu58@gmail.com
}

\begin{abstract}
In present days, real-time monitoring of the physical condition of the patients is one of the major challenges faced by hospital authorities. In this paper, we have given a solution, to face this challenge. Here, we have implemented Patient monitoring system which will be one of the major improvements in the hospitals. All the hospitals today have the monitoring systems which are wired. Also human intervention is needed frequently for critical patients. In this paper we propose a PMS (Patient Monitoring System) which can be operated wirelessly. This wireless patient monitoring system in this paper measures heartbeat, body temperature and percentage of oxygen in blood. This paper discusses about the design and development of a low cost apparatus which uses GSM technology for monitoring the health condition of the patient.
\end{abstract}

Keywords: PMS, GSM modem, SMS.

\section{Introduction}

Giving care and health assistance to the bed ridden patients at critical stages with advanced medical facilities have become one of the major problems in the modern hectic world. In hospitals where a large number of patients whose physical conditions have to be monitored frequently as a part of diagnostic procedure, the need for a cost effective and fast responding alert mechanism is inevitable. Proper implementation of such systems can provide timely warnings to the medical staffs and doctors and their service can be activated in case of medical emergencies. Present-day systems use sensors that are hardwired to a PC next to the bed. The use of sensors detects the conditions of the patient and the data is collected and transferred using a microcontroller. Doctors and nurses need to visit the patient frequently to examine his/her current condition. In addition to this, use of multiple microcontroller based intelligent system provide high level applicability in hospitals where a large number of patients have to be frequently monitored. For this, here we use the idea of network technology with wireless applicability, providing each patient a unique ID by which the doctor can easily identify the patient and his/her current status of health parameters. Using the proposed system, data can be sent wirelessly to the Central Patient Monitoring System (CPMS), allowing continuous monitoring of the patient. Contributing accuracy in measurements and providing security in proper alert mechanism give this system a higher level of customer satisfaction and low cost implementation in hospitals. Thus the patient can engage in his daily activities in a comfortable atmosphere where distractions of hardwired sensors are not present. Physiological monitoring hardware can be easily implemented using simple interfaces of the sensors with a Microcontroller and can effectively be used for healthcare monitoring. This will allow development of such low cost devices based on natural human-computer interfaces.
The system we proposed here is efficient in monitoring the different physical parameters of much number of bedridden patients and then in alerting the concerned medical authorities if these parameters bounce above its predefined critical values. Thus remote monitoring and control refers to a field of industrial automation that is entering a new era with the development of wireless sensing devices.

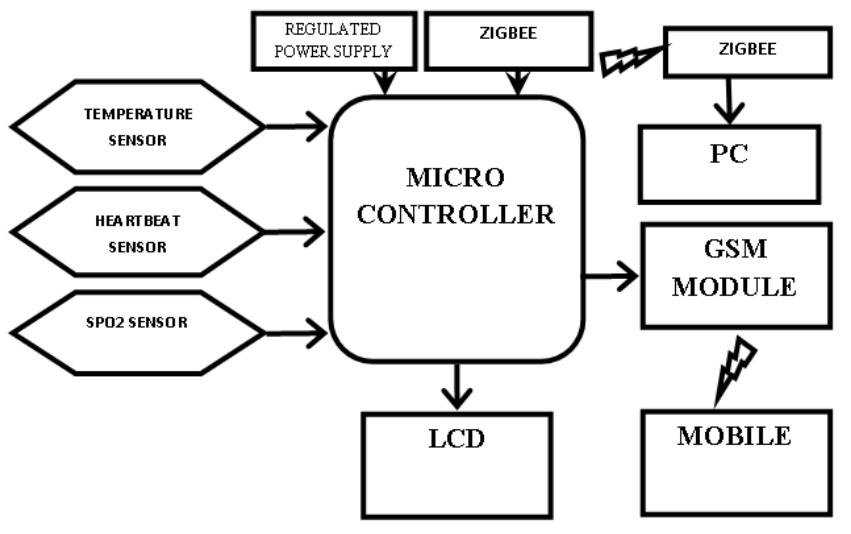

Figure 1: Block Diagram of PMS

\section{Related work}

As a part of the case study related to the various patient monitoring systems, we found that although there are many products of Patient Monitoring System in the market, few of them implemented active network technology and used mobile interface for the alert mechanism. PMS in [1] deals with the constant monitoring of health parameters using a palm-top like device and informing the service providers when ambulating conditions arise. It acts like a point-topoint system. For multiple patients in hospitals, keeping one such system for each patient will become costlier. In [2] the proposed system has the ability to measure various physical parameters of different patients, but it uses the internet facility for conveying the status of patients to the authorities. Paper [3] mainly deals with the software aspects of 
designing a system which can access the data base which include various health parameters of the patients.

\section{System design and its working}

In our proposed system we use various sensors to continuously monitor critical parameters of the patients and report to the Doctors or Nurses in attendance for timely response in case of critical situations. Our PMS has the following basic components.

- Various sensors attached to the body of the patient.

- Microcontroller for analog signal interface

- Wireless transmitting and receiving system for data transfer.

- A Central Patient Monitoring System (CPMS) observing unit basically a PC.

The sensors are attached to the body of the patients without causing any discomfort to them. Here, we monitor the important physical parameters like body temperature, percentage of oxygen in blood, heart beat rate using the sensors which are readily available. Thus the analog values that are sensed by the different sensors are then given to a microcontroller attached with it.

The microcontroller processes these analog signal values of health parameters separately and converts it to digital values using ADC converter. Now, the digitalized values are sent to the CPMS. At any time any of the doctors or nurses can log on the CPMS and check the history of the observed critical parameters of any of the patient attached to the network. When ever the sensors detect critical values, the GSM modem automatically sends the detected values to doctor's mobile via SMS. Doctor can reply back to the patient via SMS giving respective suggestions, which will be displayed on the screen.

\subsection{Internal working:}

The sensors are connected to the controller. The data detected by the sensors will be sent to the controller. Here the output of sensor is analog. So, in order to convert the analog output into digital, $\mathrm{ADC}$ is used. ADC converts analog data to digital data and this data is sent in the form of an SMS to doctor's mobile, using GSM modem. Once the doctor receives the message and checks the abnormal condition of the patient, he can suggest medicines through SMS, which will be displayed on LCD, which is connected to the controller.

\section{Flow chart}

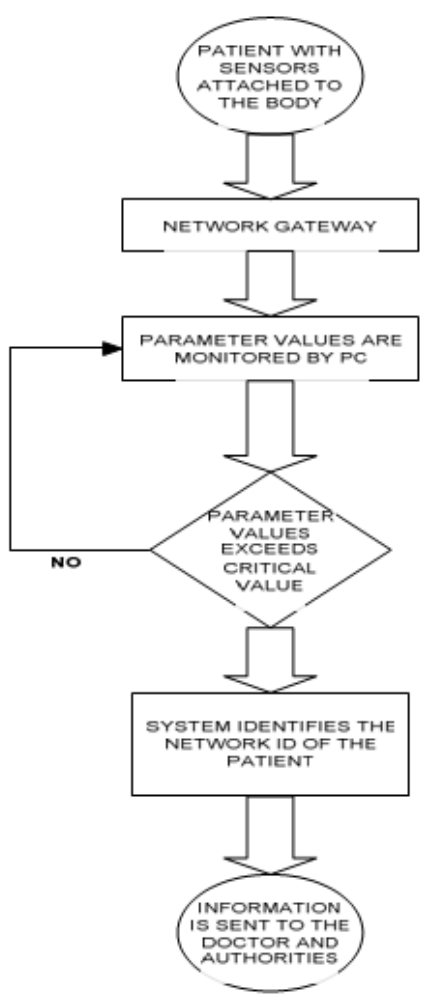

Figure 2. PMS Algorithm

\section{Modules}

In Patient Monitoring System, we use the following technologies and methodologies which will provide an active and user-friendly environment for the working of the system:

- Power supply circuit

- Micro Controller

- GSM modem

- ZIGBEE

- Heart beat sensor

- Temperature sensor

- $\mathrm{SPO} 2$ sensor

Each technology we used is discussed in detail below:

\subsection{Power supply circuit:}

The general power supply in India is $230 \mathrm{v}$ AC. But, we need only $3.3 \mathrm{v}$ DC supply for the LPC2148. Hence, we need to convert this $230 \mathrm{v}$ AC into $3.3 \mathrm{v}$ DC by using a simple circuit. This circuit consists of transformer, bridge rectifier, and capacitor and voltage regulator. First the $230 \mathrm{v}$ AC power supply is given as input to the step down transformer (120 )which step downs the 230v AC into $12 \mathrm{v}$ AC and from there we send $12 \mathrm{v} A C$ as an input to the bridge rectifier, the bridge rectifier converts the $12 \mathrm{v}$ ac into a pulsating $12 \mathrm{v}$ DC (still contains some AC components in it). Since the output of the bridge rectifier is not pure $12 \mathrm{v}$ DC we need a filter to filter all the remaining $\mathrm{AC}$ components so we are using capacitor as a filter. The $12 \mathrm{v}$ DC (pulsating) is sent to the capacitor (1000uf) it charges (like it in takes) whenever it finds the AC 
components and sends the DC components away from it. Then the output of the capacitor is pure $12 \mathrm{v}$ DC. Since we require only $3.3 \mathrm{v}$ DC then send $12 \mathrm{v}$ DC into a voltage regulator (LM317) which regulates the $12 \mathrm{v}$ DC into $3.3 \mathrm{v} \mathrm{DC}$ which is the exact voltage supply required for LPC2148 controller. By this procedure, we are converting the output voltage to our desired voltage. The desired voltage is given to the VCC (pin) \& VGND (pin) of LPC2148 microcontroller.

\subsection{Micro Controller:}

The controller which has been used here is LPC2148. LPC2148 microcontrollers are based on a 32-bit ARM7TDMI-S CPU with real-time emulation and embedded trace support that combine microcontrollers with high-speed flash memory ranging from $32 \mathrm{kB}$ to $512 \mathrm{kB}$. A 128-bit wide memory interface and unique accelerator architecture enable 32-bit code execution at the maximum clock rate. For critical code size applications, the alternative 16-bit Thumb mode reduces code by more than $30 \%$ with minimal performance penalty. Due to their tiny size and low power consumption, LPC2141/42/44/46/48 are ideal for applications where miniaturization is a key requirement, such as access control and point-of-sale. Serial communications interfaces ranging from a USB 2.0 Full-speed device, multiple UARTs, SPI, SSP to I2C-bus and on-chip SRAM of $8 \mathrm{kB}$ up to $40 \mathrm{kB}$, make these devices very well suited for communication gateways and protocol converters, soft modems, voice recognition and low end imaging, providing both large buffer size and high processing power. Various 32-bit timers, single or dual 10-bit ADCs, 10-bit DAC, PWM channels and 45 fast GPIO lines with up to nine edge or level sensitive external interrupt pins make these microcontrollers suitable for industrial control and medical systems.

\subsection{GSM:}

A GSM modem is used to alert the caretakers when there is a abrupt change in the measured parameters.

GSM (Global System for Mobile communication) is a digital mobile telephone system that is widely used in all parts of the world. GSM uses a variation of Time Division Multiple Access (TDMA) and is the most widely used of the three digital wireless telephone technologies (TDMA, GSM, and CDMA). GSM digitizes and compresses data, then sends it down a channel with two other streams of user data, each in its own time slot. It operates at either the $900 \mathrm{MHz}$ or 1,800 $\mathrm{MHz}$ frequency band.

\subsection{Zigbee:}

The low cost allows the technology to be widely deployed in wireless control and monitoring applications. Low powerusage allows longer life with smaller batteries. ZigBee is a specification for a suite of high level communication protocols using small, low-power digital radios based on an IEEE 802 standard. ZigBee is targeted at applications that require a low data rate, long battery life, and secure networking. ZigBee has a defined rate of $250 \mathrm{kbit} / \mathrm{s}$, best suited for periodic or intermittent data or a single signal transmission from a sensor or input device.

\subsection{Heart Rate:}

Heart rate is the number of heartbeats recorded per minute typically recorded as Beats per Minute (BPM) as in [7]. In the proposed system, we make use of a technique called Photoplethysmography (PPG). PPG is a simple and low cost optical technique that can be used to detect the blood volume changes in the micro vascular bed of tissues. In this technique, a bright led and a LDR is employed to detect the blood flow at the finger tip or any other peripheral part of the body. The light from the bright led gets reflected from the tissues in the body parts and the amount of light reflected determines the volume of blood flowing. If more blood flows through it, more light is reflected back.

We have to amplify the signal and remove the unwanted noise signals. For this purpose we make use of operational amplifiers, LM358. The circuit is shown below in Fig. 4.

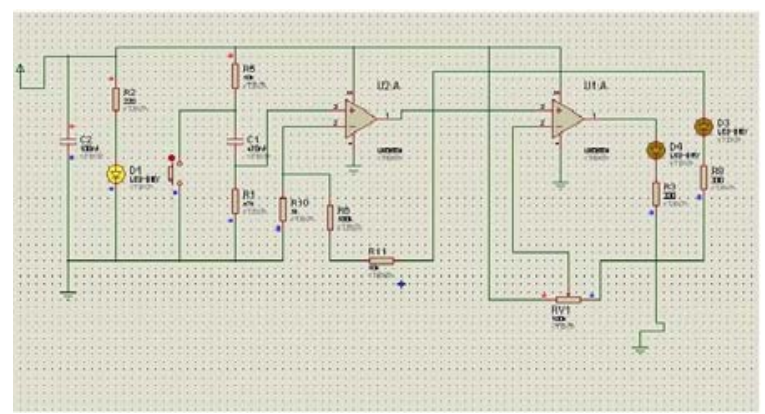

Figure 4. Noise Filtering Circuit

\subsection{Body Temperature:}

Temperature sensors in the medical field have been used from time immemorial to measure the body temperature and monitor the medical condition of patients. With a temperature sensor attached to the body of the patients, measurement of absolute temperature of the patient will be accurate, and the system allows for continuous monitoring of a patient's differential change in temperature.

The LM35 series are precision integrated-circuit temperature sensors, whose output voltage is linearly proportional to the Celsius (Centigrade) temperature. The LM35 thus has an advantage over linear temperature sensors calibrated in ${ }^{\circ}$ Kelvin, as the user is not required to subtract a large constant voltage from its output to obtain convenient Centigrade scaling. The LM35 does not require any external calibration or trimming to provide typical accuracies of $\pm / 4^{\circ} \mathrm{C}$ at room temperature and $\pm / 4^{\circ} \mathrm{C}$ over a full -55 to $+150^{\circ} \mathrm{C}$ temperature range. Low cost is assured by trimming and calibration at the wafer level. The LM35's low output impedance, linear output, and precise inherent calibration make interfacing to readout or control circuitry especially easy. It can be used with single power supplies, or with plus and minus supplies. As it draws only $60 \mu \mathrm{A}$ from its supply, it has very low selfheating, less than $0.1^{\circ} \mathrm{C}$ in still air. The LM35 is rated to operate over a $-55^{\circ}$ to $+150^{\circ} \mathrm{C}$ temperature range.

\section{Features:}

- Calibrated directly in ${ }^{\circ}$ Celsius (Centigrade)

- Linear $+10.0 \mathrm{mV} /{ }^{\circ} \mathrm{C}$ scale factor

- $\quad 0.5^{\circ} \mathrm{C}$ accuracy guaranteeable (at $+25^{\circ} \mathrm{C}$ ) 
- $\quad$ Rated for full $-55^{\circ}$ to $+150^{\circ} \mathrm{C}$ range

- Suitable for remote applications

- Low cost due to wafer-level trimming

- Operates from 4 to 30 volts

- Less than $60 \mu \mathrm{A}$ current drain

- $\quad$ Low self-heating, $0.08^{\circ} \mathrm{C}$ in still air

- Nonlinearity only

- $\quad \pm / 4^{\circ} \mathrm{C}$ typical

- $\quad$ Low impedance output, $0.1 \Omega$ for $1 \mathrm{~mA}$ load

\subsection{SPO2 sensor:}

The body's need for oxygen is certain. Its availability at a tissue level is sometimes in doubt. Blood gas measurements provide critical information regarding oxygenation, ventilation, and acid-base status. However, these measurements only provide a snapshot of the patient's condition taken at the time that the blood sample was drawn. It is well known that oxygenation can change very quickly. In the absence of continuous oxygenation monitoring, these changes may go undetected until it is too late. Pulse oximeters measure blood oxygen saturation noninvasively and continuously.

A blood-oxygen saturation reading indicates the percentage of hemoglobin molecules in the arterial blood which are saturated with oxygen. The reading may be referred to as $\mathrm{SaO}$. Readings vary from 0 to $100 \%$. Normal readings in a healthy adult, however, range from $94 \%$ to $100 \%$. The term $\mathrm{SpO} 2$ means the $\mathrm{SaO}$ measurement determined by pulse oximetry.

\subsubsection{How Does Pulse Oximetry Work?}

Within the Sp02 sensor, light emitting diodes shine red and infrared light through the tissue. Most sensors work on extremities such as a finger, toe or ear. The blood, tissue and bone at the application site absorb much of the light. However, some light passes through the extremity. A lightsensitive detector opposite the light source receives it.

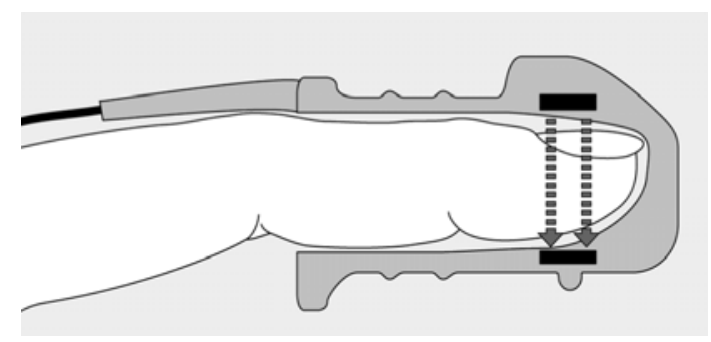

Fig 3: SPO2 sensor

The sensor measures the amount of red and infrared light received by the detector and calculates the amount absorbed. Much of it is absorbed by tissue, bone and venous blood, but these amounts do not change dramatically over short periods of time. The amount of arterial blood does change over short periods of time due to pulsation (although there is some constant level of arterial blood). Because the arterial blood is usually the only light absorbing component which is changing over short periods of time, it can be isolated from the other components.

\section{Experimental setup}

The modem which we are using is GPRS/GSM modem. GSM/GPRS modem can be used to send messages and also make a call through computer. HyperTerminal can be used to control the modem. For interfacing HyperTerminal with modem there are some steps which we should follow.

\subsection{Setting up GSM Modem}

GSM/GPRS modem is connected to the computer using a RS-232 cable. It is connected to the serial port of the computer. The GSM modem will map itself as a COM serial port in the computer as in [9].

\subsection{HyperTerminal Configuration}

- On the Windows Start menu, select the Run dialog box.

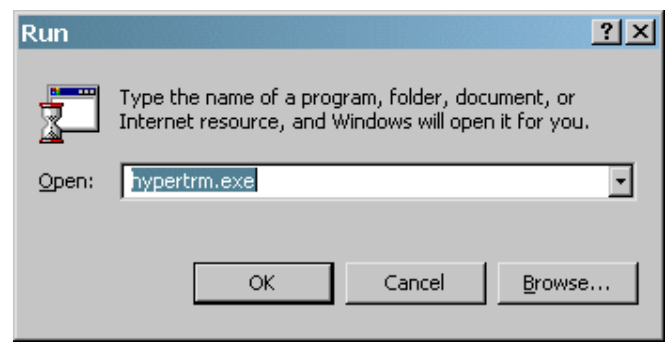

Figure. 5. The Windows Run Dialog Box

- Type hypertrm.exe in the Open field to open the Connection Description screen as shown in Fig.5

- On the Connection Description screen, for Name, "Cisco" is typed and an icon is selected for the definition. The Connect to dialog box appears.

- The primary COM port is selected for the Connect. The COM Properties dialog appears.

- On the COM Properties dialog box, the following selections are made as shown in the Fig 6.

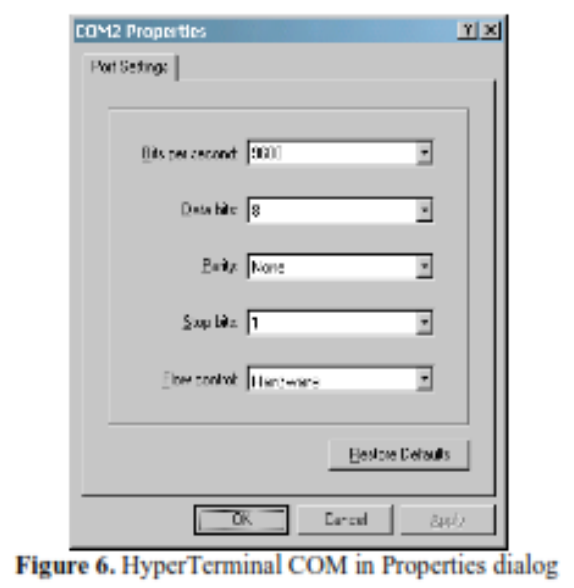

Bits per sec: 115200

Data bits: 8

Parity: none

Stop bits: 1

Flow control: Hardware 


\subsection{Setting up initially by AT Commands}

We are now ready to start working with AT commands to setup and check the status of the GSM modem as shown in [10].

Returns a "OK" to confirm that modem is working.

$$
\begin{aligned}
& \text { AT }+ \text { CREG A " } 0 \text { " or " } 1 \text { " reply confirms that modem is } \\
& \text { connected to GSM network. }
\end{aligned}
$$

\subsection{Sending SMS through AT Commands.}

To format SMS as a TEXT message.

\section{Future work}

The scalability of the proposed system opens for a wide range of applicability in Multispecialty hospitals where many number of critical care units is present. The scalability is achieved by the networking facility which provides multiple Sensor-Microcontroller modules to send data consecutively to the gateway attached to the PC. Along with this, expanding the project to allow two way communications between doctors and patients, will be beneficiary in many cases where patient needs to communicate directly to the doctor. This will allow doctors to send messages to the patients, and thus make the consultation and service provision more transparent and effective.

\section{Conclusion}

By implementing active network based wireless technology with sensor-microcontroller module, our proposed NWSPMS which is responsible for monitoring the health of many number of patients in the same critical unit. The system proposed above is the cheapest system that can be used for this purpose. One of the important beneficiaries of the system is the doctors who can monitor the physical and medical conditions of their patients from any part of the world and thus he can give instructions to others to attend to the patient. Thus the proposed system is in other words an e-hospital system, where the doctor and the patient can attend to many patients at a time .Since it is a continuous monitoring system, the doctor would be able to give clear and best instructions within the least time. By applying this scalability of the proposed system in the rural areas where healthcare facilities are most needed, the system can act as bride between the rural patient and the urban medical service provider. The further work in this topic will focus on the possibility of an effective and cheap home automation system which can be realized by reducing the number of sensor microcontroller module to a single unit.

\section{References}

[1] Aart Van Halteren, Richard Bults, Katarzyna Wac, Dimitri Konstantas, Ing Widya, Nicolay Dokovsky, George Koprinkov, Val Jones, Rainer Herzog, "Mobile Patient Monitoring: Te MobiHealth System", Te Journal on Information Technology in Healthcare 2004; 2(5): 365-373
[2] Emil Jovanov, Dejan Raskovic, John Price, John Chapman, Anthony Moore, Abhishek Krishnamurthy, "Patient Monitoring Using Personal Area Networks of Wireless Intelligent Sensors"

[3] Ning Li, Sagar Kotak, Amogh Badwe, "Patient Monitoring System"

[4] Details of Blood Pressure sensor from fingers, Available on www.coolcircuit.com

[5] Details of ECG sensor, Available on www.sensorsmag.com and www.swharden.com

[6] Datasheets of PIC 16f877A, MAX232, LM358, LM324, LM 335.Available on www.alldatasheet.com

[7] Information about heart rate monitoring: www.heartmoniters.com

[8] Information on MAX 232 IC

[9] Information on serial port

[10] Modem PC configuration through AT

\section{Author profile}

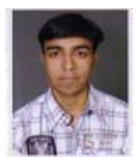

Chanakya Mothukuri received B-tech degreein Electronics and communications engineering from Balaji institute of Technology and science in 20052009. M-tech degree in Embedded systems from CVSR College of engineering in 2010-2013.

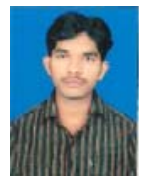

K CH Prathap Kumar M was born in Ongole, Andhra Pradesh, India, in 1987. He received the B.Tech degree in Electronics and Communication Engineering from JNTU, Anantapur, in 2009. He received the Masters degree in VLSI System Design from HITS, Hindustan University, Chennai in 2011. His research interest includes Digital Image Processing and architecture designs for probabilistic CMOS technology. 